\section{JAPAN-TSUNAMI}

\section{Rasanter Anstieg von Herzkrankheiten in den Folgewochen}

— Erdbeben und Tsunami im März 2011 in Japan kosteten etwa 20000 Menschen das Leben. Doch Stress und Chaos setzen den Menschen auch in der Folgezeit zu: Morbidität und vermutlich auch Mortalität blieben noch über einige Wochen erhöht. Insbesondere Herzinfarkte, Schlaganfälle, Fälle von dekompensierter Herzinsuffizienz und Pneumonien häuften sich, berichtete Dr. H. Shimokawa von der Universitätskli-

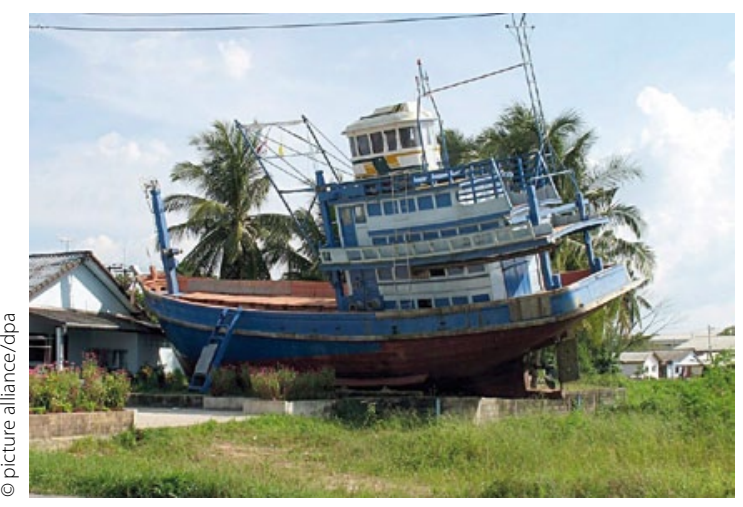

nik in Sendai, auf dem ESC-Kongress. Die Autoren hatten die Ambulanzeinsätze in den Wochen vor und nach der Naturkatastrophe ausgewertet. Dabei zeigte z. B. der Schlaganfall einen zweiten Gipfel zum Zeitpunkt des heftigsten Nachbebens Wochen nach dem Tsunami.

Die Ursachen liegen auf der Hand: Zum Anstieg der Krankheiten dürften stressund angstbedingte Aktivierungen des Sympathikus nebst Blutdruckanstieg eine Rolle gespielt haben, so der Referent. Auch der Zusammenbruch der Infrastruktur, der medizinischen Versorgung und die Unterbrechung von Medikationen haben eine Rolle gespielt.

VERONIKA SCHLIMPERT .

Nicht alle Folgen des Tsunami waren auf den ersten Blick sichtbar.
BULLENSTARKES HERZ

\section{Kultgetränk stärkt Kontraktionskraft}

- Millionen Jugendliche schwören auf Energydrinks, um für die lange Partynacht länger fit zu bleiben. Doch was bewirkt die koffein- und taurinhaltige Powerbrause eigentlich am Herzen?

Italienische Kardiologen machten sich jetzt mit modernster Ultraschalltechnik in einer Studie mit 35 gesunden jungen Teilnehmern auf die Suche. Das Ergebnis: Eine Stunde nach Zufuhr des Energie-Trunkes (auf den beliebten Vodka-Zusatz wurde verzichtet) zeigten sich wichtige Parameter der Herzfunktion gebessert, insbesondere die energetisch wichtige Torsion beider Ventrikel. Die linksventrikuläre Ejektionsfraktion erhöhte sich um 5\%, der diastolische Blutdruck um 6\%, berichtete Dr. M. Cameli aus Siena. Nach Konsum von Fruchtsaft wurden diese Effekte nicht beobachtet.

SARAH PAMPEL

\title{
Das Herz in der Hose
}

In München beim Europäischen Kardiologenkongress. Beim Rundgang über die Ausstellung wurde ich mit folgendem überraschenden Werbeslogan konfrontiert: Ein neues Lebensgefühl dank HerzHose.

Bisher war mir die Konnotation von Herz und Hose nur als Zustandsbeschreibung bekannt, nämlich dann, wenn ersteres in letzteres gerutscht ist. Zunächst dachte ich, es handele sich um ein modisches Beinkleid, welches alle Kardiologen tragen sollten, ähnlich wie die Herz-Krawatte, um bereits mit dem äußeren Erscheinungsbild seine Zugehörigkeit zu dieser erlauchten Berufsgruppe signalisieren zu können. Dass der Erwerb einer solchen Hose darüber hinaus zugleich mit einer Spende für einen guten kardiologischen Zweck verbunden sein würde, war für mich eigentlich selbstverständlich. Aber der stolze Preis von 90000 Euro machte mich dann doch etwas stutzig. Auch war mir bisher kein Kollege mit einem solchen "kardialen“ Outfit begegnet. Irritierend war auch das besondere modische Design - es handelt sich nämlich um eine sehr dicke blaue Hose, die bis über den Nabel reicht, sodass der Träger wie das Ergebnis einer modischen Verirrung aussehen dürfte. So regten sich bei mir dann doch Zweifel, ob meine primäre Vermutung richtig sei.

Bei genauerem Hinsehen klärte sich die Sache dann doch schnell. Es geht bei dieser "Herzhose" nämlich um eine neue Behandlungsmethode für die Herzschwäche; denn nachdem bei herzinsuffizienten Patienten mittlerweile alle Rezeptoren am Herzmuskel mit irgendwelchen Pharmaka besetzt sein dürften und im Binnenraum des Herzens nach Implantation verschiedener Devices wie biventrikulärem Schrittmacher und automatischem Defibrillator auch kaum noch Platz ist für neue Sonden, musste man sich ja etwas neues ausdenken, nämlich etwas, was außerhalb des Herzens angreift, also quasi ein Zusatzherz. Und was liegt da näher als ein solches in eine Hose zu stecken!

Und wie wirkt diese Herz- Hose? Bei jedem Herzschlag entwickelt das pulsierende Beinkleid eine kontrollierte Schubkraft und hilft so dem schwachen Herzen ähnlich wie ein E-Bike. Es ist also das genaue Gegenteil von einem leblosen Beinkleid, auch tote Hose genannt. Mit seiner Hilfe wird das Blut künstlich in Wallung gebracht. Kurzum, es handelt sich in der Tat um einen revolutionären Fortschritt in der Hose oder besser gesagt mit der Hose. Entscheidend ist aber nicht die Hose selbst, sondern das Herz, das in ihr schlägt.

Dr. Peter Stiefelhagen 